OPEN ACCESS

Edited by:

Tao Xu,

Anhui Medical University, China

Reviewed by:

Zhao Kun,

Beijing Cancer Hospital, China Hongzhou Cui,

First Hospital of Shanxi Medical University, China

*Correspondence: Jianzhong Zhang rmzjz@126.com

Specialty section:

This article was submitted to Inflammation Pharmacology,

a section of the journal

Frontiers in Pharmacology

Received: 12 January 2021 Accepted: 10 February 2021 Published: 22 March 2021

Citation:

Wu X, Zhao Y, Gu Y, Li K, Wang X and Zhang J (2021) Interferon-Lambda 1 Inhibits Staphylococcus aureus

Colonization in Human

Primary Keratinocytes.

Front. Pharmacol. 12:652302. doi: 10.3389/fphar.2021.652302

\section{Interferon-Lambda 1 Inhibits Staphylococcus aureus Colonization in Human Primary Keratinocytes}

\author{
Xia $W u^{1}$, Yan Zhao ${ }^{1}$, Ying $\mathrm{Gu}^{2}$, Kun $\mathrm{Li}^{1}$, Xiaojie Wang ${ }^{1}$ and Jianzhong Zhang ${ }^{1 *}$ \\ ${ }^{1}$ Department of Dermatology, Peking University People's Hospital, Beijing, China, ${ }^{2}$ Department of Dermatology, Beijing Children's \\ Hospital, Capital Medical University, National Center for Children's Health, Beijing, China
}

Atopic dermatitis (AD) is a common inflammatory skin disease. Staphylococcus aureus ( $S$. aureus) colonization in skin lesions occurs in approximately $70 \%$ of AD patients. It has been found that IFN- $\lambda 1$ can inhibit the colonization of $S$. aureus in normal human nasal mucosa. IFN- $\lambda 1$ can increase IL-28RA in infected human keratinocytes. In this study, we found that IFN- $\lambda 1$ can increase mRNA expression of FLG and antimicrobial peptides (AMPs) and inhibit TSLP mRNA expression in infected human keratinocytes. IFN- $\lambda 1$ can increase intracellular ROS level, decrease STAT1 phosphorylation, and inhibit the colonization of $S$. aureus in human primary keratinocytes. These effects were attenuated by knocking-down IL-28R and NADPH oxidase inhibitor, suggesting that this function was mediated by JAKSTAT1 signaling pathway. These results suggest that IFN- $\lambda 1$ might have an inhibitory effect on $S$. aureus colonization in $\mathrm{AD}$ lesions. Our findings might have potential value in the treatment for $A D$.

Keywords: interferon-lambda 1, Staphylococcus aureus, atopic dermatitis, keratinocyte, reactive oxygen species, signal transducerand activator of transcription 1

\section{INTRODUCTION}

Atopic dermatitis is a common inflammatory skin disorder. The prevalence of $\mathrm{AD}$ is approximately $30 \%$ in children and 10\% in adults (Bin et al., 2014; Langan et al., 2020). AD is characterized by eczematous lesions with severe itching and often associated with allergic rhino-conjunctivitis, food allergy and asthma (Alduraywish et al., 2016; Matsumoto et al., 2020). Skin barrier defect, immune dysregulation, environmental factors and microbial dysbiosis contribute to the pathogenesis of $\mathrm{AD}$ (Orfali et al., 2019; Ahn et al., 2020). AD is a type 2 inflammatory disease, with increased Th2 cytokine levels, such as interleukin (IL)-4, IL-5, and IL-13 (Eyerich and Novak, 2013). In the acute phase, Th2 and Th2 2 responses are amplified. In the chronic phase, however, Th1 and Th17 are also activated (Weidinger and Novak, 2016). Increased Staphylococcus aureus skin colonization is another hallmark of AD, which is often associated with disease severity and exacerbation (Byrd et al., 2017; Tay et al., 2020). Specific immunoglobulin E against S. aureus enterotoxin (SE-IgE) has been found in AD patients (Motala et al., 1986). S. aureus was found in approximately 70\% AD lesions and 39\% non-lesional skin (Totte et al., 2016). It has been reported that $S$. aureus can impair barrier function, inhibit antimicrobial peptides (AMP) production, and promote viral loads of HSV-1 and Th2 response (Bin et al., 2012; Geoghegan et al., 2018). Impairment of bacterial clearance might exacerbate the inflammation.

Interferon- lambda (IFN- $\lambda$ ) is a novel type III IFNs, including IFN- $\lambda 1$ (also known as interleukin (IL)-29), IFN- $\lambda 2$ (IL-28A), IFN- $\lambda 3$ (IL-28B) and IFN- $\lambda 4$ in humans (Broggi et al., 2020). Both type III and type I IFNs $(\alpha / \beta)$ have antiviral function. Moreover, the type III IFNs are members of the 
IL-10 related cytokine family (Kotenko et al., 2003; Lazear et al., 2015). IFN- $\lambda$ has a different conformation from type I IFNs $(\alpha / \beta)$ and interacts with a heterodimeric receptor (IFN- $\lambda \mathrm{R}$ ) composed of a unique ligand-binding chain IL-28 receptor A (IL-28RA) and an accessory chain, IL-10 receptor B (IL-10RB), which is shared with the IL-10 receptor and other IL-10-related cytokine receptors (Kotenko et al., 2003). After binding to its receptor, IFN- $\lambda 1$ activated the Janus kinase-signal transducer and activator of transcription (JAK-STAT) signaling pathway (Sheppard et al., 2003; Zitzmann et al., 2006; Maher et al., 2008). The predominant expression of IFN- $\lambda \mathrm{R}$ on respiratory and gastrointestinal epithelial cells (Sommereyns et al., 2008; Banos-Lara Mdel et al., 2015), keratinocytes (KCs) (Witte et al., 2009) and hepatocytes (Marukian et al., 2011) allows strong antiviral effects at local barrier sites without activating a systemic proinflammatory immune response.

IFN- $\lambda s$ have vital functions in defensing against colonization of gram-positive bacteria on epithelial tissues and nasal tissues (Bierne et al., 2012; Lan et al., 2019). Compared with IL-28R ${ }^{-/-}$ mutant mice, the $S$. aureus colonization of wild-type mice had increased after nasal infection with influenza virus, indicating that IL-28R was involved in the colonization of $S$. aureus (Planet et al., 2016). IFN- $\lambda 1$ was found in the nasal tissue of patients associated with chronic rhinosinusitis with nasal polyps after $S$. aureus infection. However, the antibacterial role was only found in healthy tissue (Lan et al., 2019). The anti-bacterial effect of IFN- $\lambda$ has been studied in nasal mucosa tissue of IL-28R ${ }^{-/-}$mouse model and in human nasal tissue. Little is known about the role of IFN- $\lambda$ in skin $S$. aureus infection. We used human keratinocyte- $S$. aureus infection model to study the role of IFN- $\lambda 1$ in the immune response to $S$. aureus skin infection. We evaluated the number of $S$. aureus colony forming units (CFU) and ROS expression in keratinocytes in the presence of IFN- $\lambda 1$, attempting to elucidate the possible mechanism of anti-bacterial effects of IFN- $\lambda 1$.

\section{MATERIALS AND METHODS}

\section{Keratinocyte Culture}

Human primary keratinocytes were cultured in keratinocyte culture medium (KCM) (CELLnTEC, CnT-07, Swiss) containing $100 \mathrm{U} / \mathrm{ml}$ (penicillin)-100 $\mu \mathrm{g} / \mathrm{ml}$ (streptomycin) (Invitrogen, 15070063, United States) and $10 \mu \mathrm{g} / \mathrm{ml}$ (gentamicin)-250 ng/ml (amphotericin B) (Invitrogen, R01510, United States) in a humidified atmosphere of $5 \% \mathrm{CO}_{2}$ and $37^{\circ} \mathrm{C}$. When keratinocytes reached $80 \%$ confluency, the culture media were changed to antibiotic-free medium for subsequent experiments.

Keratinocytes were fixed with $4 \%$ paraformaldehyde for $15 \mathrm{~min}$ at room temperature, then permeabilized with $0.1 \%$ Triton X-100. Anti-Cytokertin 5 antibody (1:100, Abcam, ab52635, United Kingdom) and anti-Cytokertin 10 antibody (1:150, Abcam, ab76318, United Kingdom) were applied to keratinocytes and incubated for $1 \mathrm{~h}$. After 3 times washing, goat anti-rabbit IgG ( $\mathrm{H}+\mathrm{L})$-CoraLite594 (red) (1:500, Proteintech, SA00013-4, United States) was applied. Images were captured on fluorescence microscope (Leica, DMi8,
Germany). DAPI (blue) was used for nuclear counterstain (Supplementary Figure S1).

\section{Cell Proliferation Assay}

The effect of IFN- $\lambda 1$ to keratinocyte proliferation was measured by Cell Counting kit (CCK-8) assay. Keratinocytes were seeded in a 96-well plate (about $3 \times 10^{3}$ cells per well). After $24 \mathrm{~h}$ incubation, IFN- $\lambda 1$ at the concentrations of $1 \mathrm{ng} / \mathrm{ml}, 10 \mathrm{ng} / \mathrm{ml}$ and $100 \mathrm{ng} / \mathrm{ml}$ (Pereprotech, AF-300-02L, United States) were added to the culture media. $10 \mu$ of TransDetect ${ }^{\circledR}$ Cell Counting Kit (CCK) (Transgen, FC101-01, China) was added to each well at the time of $0,24,48$, and $72 \mathrm{~h}$, respectively. After $1 \mathrm{~h}$ incubation, the absorbance at $450 \mathrm{~nm}$ was measured by the Multiskan $^{\mathrm{TM}}$ FC Microplate Photometer (Thermo Scientific, 51119180, Belgium). Cell colony formation assay was also performed to verify the effect of IFN- $\lambda 1$ on keratinocyte proliferation. Keratinocytes were planted to 6 -well plate at 2,000 cells each well and cultured with various concentration of IFN- $\lambda 1$ for 15 days. Phosphate buffered saline containing $5 \%$ trehalose (5\% trehalose-PBS) (MultiSciences, 79-PD0021, China) was used as negative control. After 15 days culture, the cells were fixed with formaldehyde, stained with $0.1 \%$ crystal violet, washed with 33\% glacial acetic acid and measured with a Multiskan FC microplate photometer (Thermo Scientific, 51119180, Belgium).

\section{Knockdown of Protein Expression by siRNA Transfection}

Three human IL-28RA siRNAs and negative control siRNA were purchased from GenePharma (Figure. S4A). According to the manufacturer's protocol, negative control siRNA (siNC), IL28RA-1 siRNA (silL28RA-1), IL28RA-2 siRNA (silL28RA2), and IL28RA-3 siRNA (silL28RA-3) were transfected into keratinocytes using Lipofectamine 3000 Transfection Reagent (Invitrogen, L3000015, United States). After $72 \mathrm{~h}$ culture, the cells were harvested for subsequent experiments. Quantitative real-time PCR (qPCR) was used to detect the mRNA level of IL28RA and western blotting was used to detect the protein level of IL-28RA.

\section{Preparation of Staphylococcus aureus}

Staphylococcus aureus strain was donated by department of laboratory, Peking University People's Hospital, which was isolated from $\mathrm{AD}$ eczematous skin lesions. S. aureus were grown in $10 \%$ sodium chloride tryptone soy broth (TSB) (Solarbio, LA3750, China) medium for $16-18 \mathrm{~h}$ at $37^{\circ} \mathrm{C}$ under agitation $(120 \mathrm{rpm})$ and collected by centrifugation at $2000 \mathrm{rpm}$ for $10 \mathrm{~min}$ at room temperature. After washing three times, $S$. aureus were resuspended in PBS (Gibco, 10010049, United States). The concentration of bacteria was measured by the Multiskan $^{\mathrm{TM}}$ FC Microplate Photometer at $600 \mathrm{~nm}$ wavelength and diluted properly before infection experiment. The bacteria samples were planted onto the mannitol salt agar (MSA) plate (Solarbio, LA 1980, China) for $24 \mathrm{~h}$ at $37^{\circ} \mathrm{C}$ (Supplementary Figure S2A). The 16s rRNA, femA, and mecA were measured by multiplex PCR with the EmeraldAmp ${ }^{\circledR}$ PCR Master Mix (Supplementary Figure S2B). 


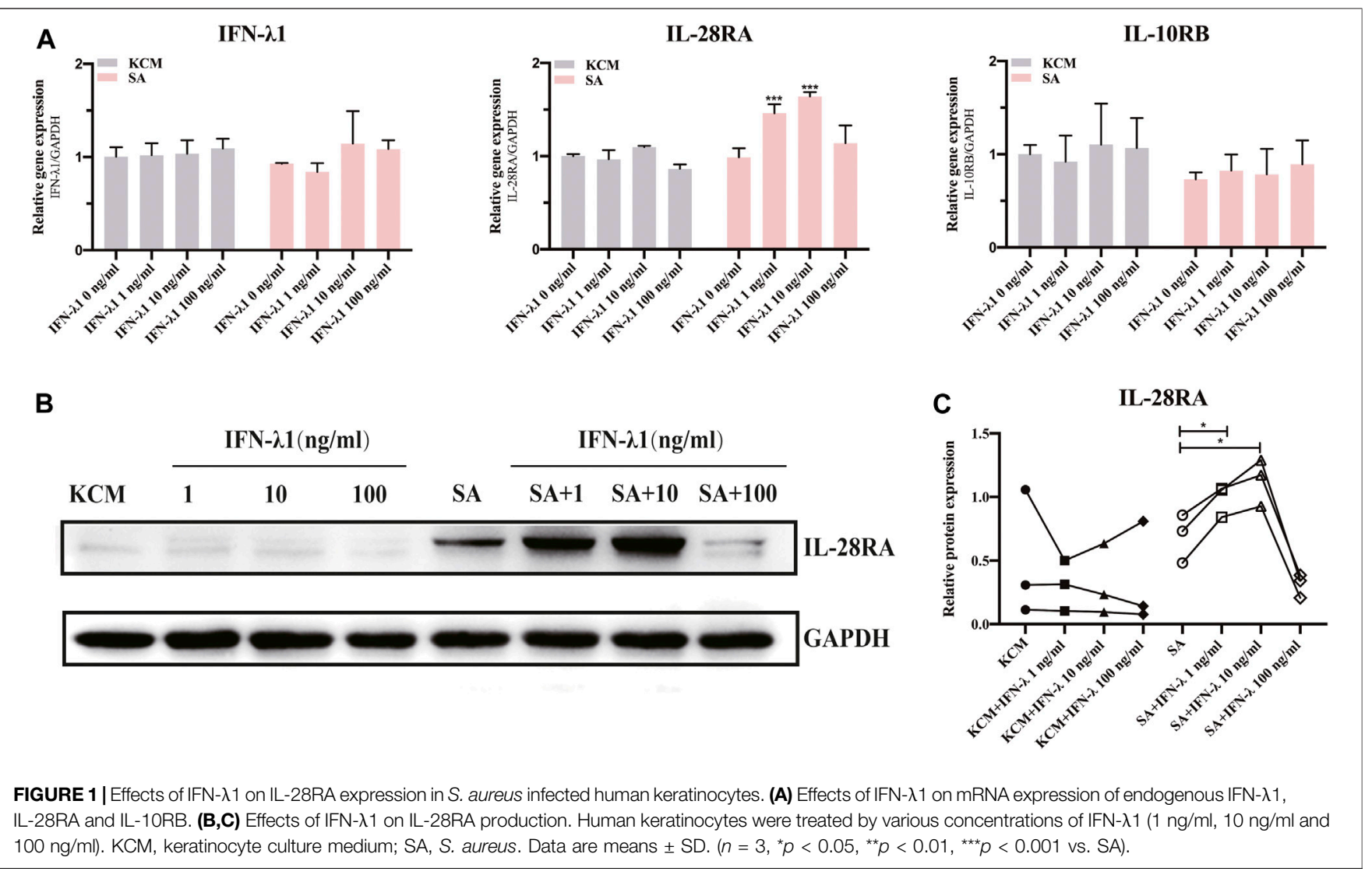

The primer sequences used in the multiplex PCRs were described in Supplementary Table S1. The sequencing of PCR products was performed by Sangon Biotech (Shanghai, China), and the resulting sequences were compared with $S$. aureus strains in Genebank by using the BLAST online program on the NCBI website (Supplementary Figure S3).

\section{Keratinocyte Infection Model}

Human primary keratinocytes were planted into the 6-well plate and incubated with IFN- $\lambda 1(1 \mathrm{ng} / \mathrm{ml}, 10 \mathrm{ng} / \mathrm{ml}$ and $100 \mathrm{ng} / \mathrm{ml}$ ) or $5 \%$ trehalose-PBS as control for $24 \mathrm{~h}$. S. aureus were added to keratinocyte culture at the ratio of 30:1 (bacteria: keratinocytes) and incubated for $3 \mathrm{~h}$ (infection model). Keratinocytes without $S$. aureus infection were also incubated in the presence of IFN- $\lambda$ (non-infection model). After incubation, the infected keratinocytes and noninfected keratinocytes were used for qPCR and western blotting.

In order to study the role of IFN- $\lambda 1$ in elimination of $S$. aureus, keratinocytes were treated with silL28RA-1 for $48 \mathrm{~h}$ or $10 \mu \mathrm{M}$ NADPH oxidase inhibitor diphenyleneiodonium chloride (DPI) $(\mathrm{APO} \times \mathrm{BIO}, \mathrm{B} 6326$, United States) for $1 \mathrm{~h} . \mathrm{IFN}-\lambda 110 \mathrm{ng} / \mathrm{ml}$ were then added to culture media and incubated for $24 \mathrm{~h}$ before $S$. aureus infection. The keratinocyte lysates were collected for detection of STAT1 phosphorylation. The intracellular ROS expression was measured by flow cytometry and fluorescence microscopy. The colony forming unit (CFU) was also evaluated.

\section{Quantitative Real-Time PCR}

Total RNA was extracted from keratinocytes by using the total RNA isolation kit (Beibei Biotechnology, 082001, China) and was reverse-transcribed by using the TransScript ${ }^{\circledR}$ All-in-one firststrand cDNA synthesis superMix for qPCR kit (Transgen, AT341, China). The mRNA expression of IFN- $\lambda 1$, IL-28RA, IL-10RB, involucrin (IVL), filaggrin (FLG), thymic stromal lymphopoietin (TSLP), human antimicrobial $\beta$-defensin (hBD) 1, hBD2, hBD3, S100 calcium binding protein A7 (S100A7), S100A8 and S100A9 was measured by qPCR, using SYBR ${ }^{\circledR}$ Premix Ex $\mathrm{Taq}^{\mathrm{TM}}$ II (Takara, RR820A, Japan) on the LightCycler 480 Instrument II (Roche Applied Science, Germany). All primers were listed in Supplementary Table S2. The housekeeping genes (GAPDH and $\beta$-actin) were used to normalize transcription and amplification variations among the samples. All primers were purchased from Sangon Biotech (Shanghai, China). Relative expression ratio was calculated using the comparative threshold cycle $(\mathrm{Ct})$ and $2^{-\Delta \Delta \mathrm{Ct}}$ method. All experiments were performed in triplicate.

\section{Western Blotting}

The treated keratinocytes were lyzed with RAPI buffer (New Cell \& Molecular Biotech, WB3100, China) containing proteinase and phosphatase inhibitor cocktail (Beyotime Biotechnology, P1045, China). Protein of each sample was separated by $10 \%$ SDS-PAGE (Biotides Biotech, WB2102, China) and transferred to polyvinylidene fluoride 
A

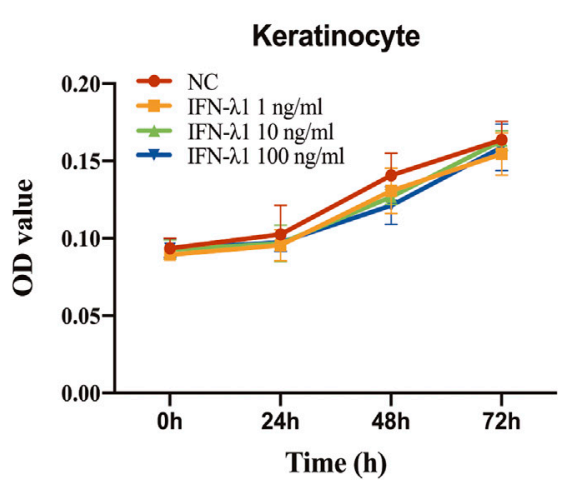

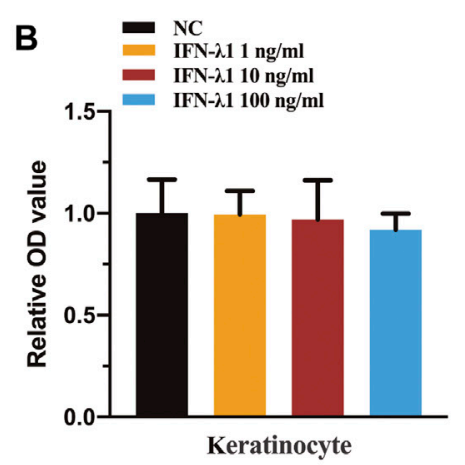

C

Keratinocyte
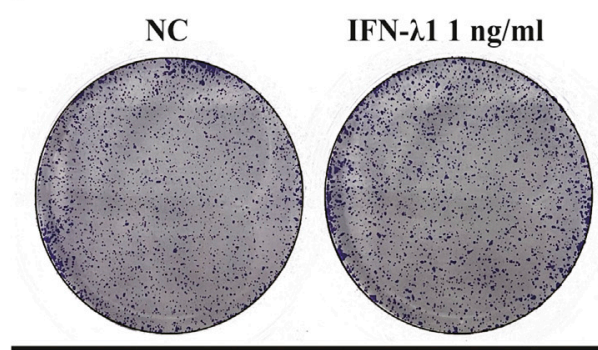

IFN- $\lambda 110 \mathrm{ng} / \mathrm{ml}$

IFN- $\lambda 1100 \mathrm{ng} / \mathrm{ml}$

D

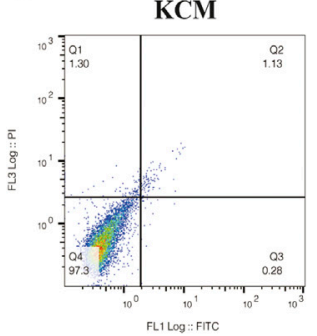

$\mathrm{KCM}+\mathrm{IFN}-\lambda 11 \mathrm{ng} / \mathrm{ml}$
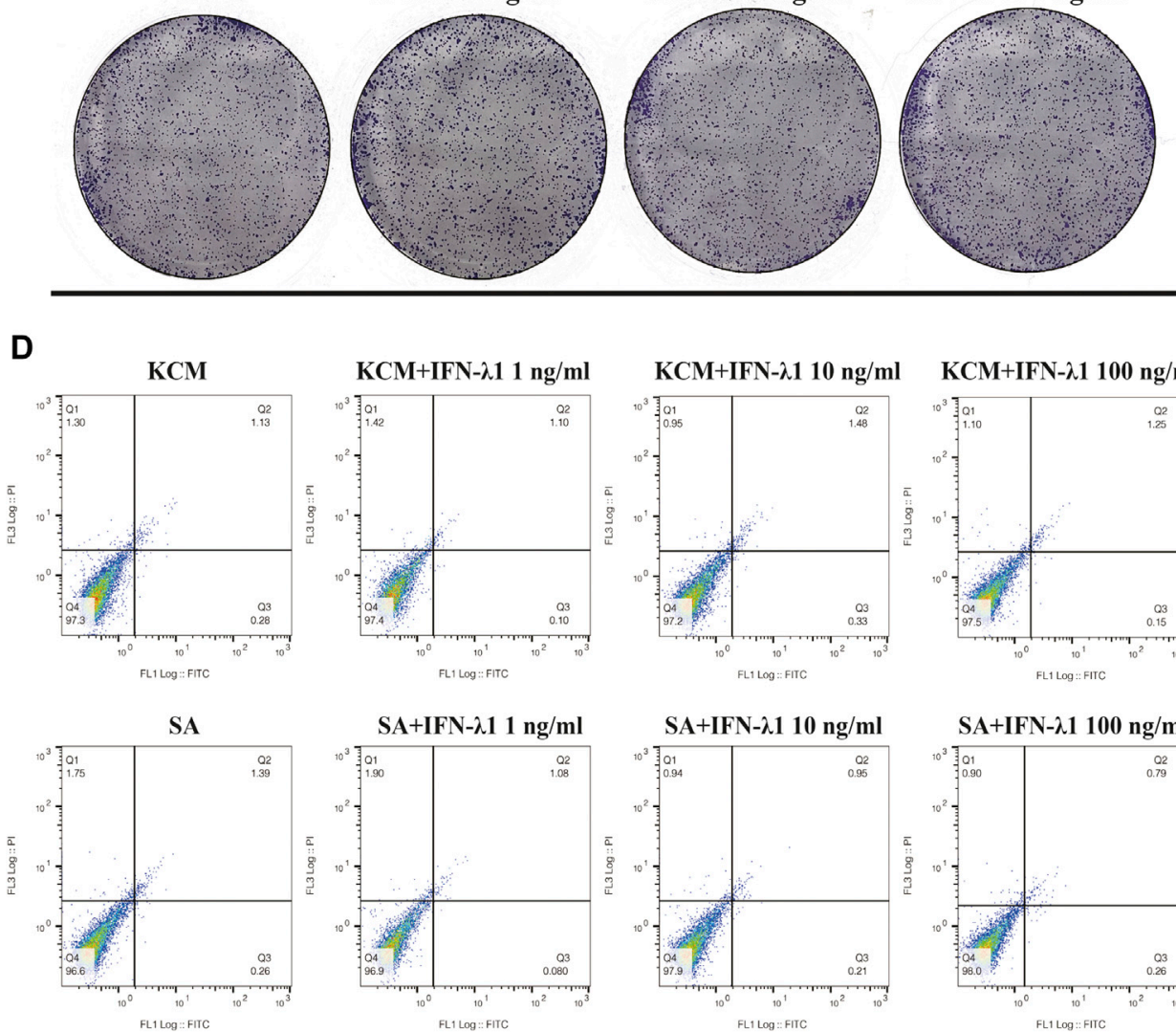

KCM+IFN- $\lambda 1100 \mathrm{ng} / \mathrm{ml}$
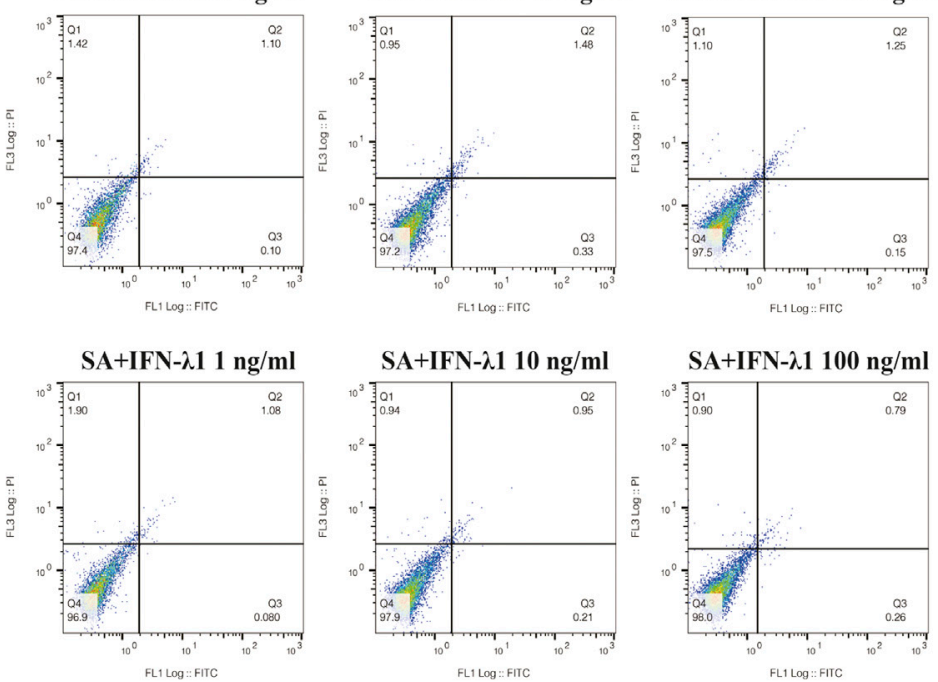

FIGURE 2 | No effect of IFN- $\lambda 1$ was found on keratinocyte proliferation and apoptosis. (A-C) Result of keratinocyte proliferation assays: (A) CCK-8 assay; (B,C) Colony-formation assay; (D) Results of keratinocyte apoptosis assay. Human keratinocytes were treated by various concentrations of IFN- $\lambda 1$ (1 ng/ml, $10 \mathrm{ng} / \mathrm{ml}$ and $100 \mathrm{ng} / \mathrm{ml})$. KCM, keratinocyte culture medium; SA, S. aureus. Data are means $\pm \mathrm{SD}$. $\left(n=3,{ }^{*} p<0.05\right)$.

membranes (EMD Millipore, ISEQ00010, United States). The primary antibodies for western blotting included antiSTAT1 (1:1,000, CST, 14995, United States), anti-phosphoSTAT1 (Tyr701) (1:1,000, CST, 9167, United States) and anti-IL-28R antibody (1:750, Abcam, ab224395, United Kingdom). The blots were then incubated with anti-rabbit IgG, HRP-linked antibody (1:3,000, CST, 7074, United States). The specific bands were observed by Tanon
Imaging System (Tanon, China) with Immobilon Western Chemiluminescent HRP Substrate (Millipore, WBKLS0500, United States). The band intensity was calculated by ImageJ software (National Institutes of Health, Bethesda, MD) compared with GAPDH (1:10,000, Proteintech, 60004-1lg, United States). The secondary antibody for GAPDH was Goat Anti-Mouse IgG H\&L (HRP) (1:9,000, Abcam, ab6789, United Kingdom). 


\section{Cell Apoptosis Assay}

Cell apoptosis was measured by TransDetect ${ }^{\circledast}$ Annexin V-FITC/ PI Cell Apoptosis Detection Kit (Transgen, FA101, China). After treatment with various concentrations of IFN- $\lambda 1$ for $24 \mathrm{~h}$ and infected by $S$. aureus, keratinocytes $\left(2 \times 10^{5}\right.$ cells $)$ were harvested, resuspended in $100 \mu \mathrm{l}$ one $\mathrm{x}$ Annexin $\mathrm{V}$ binding buffer and incubated with $5 \mu \mathrm{l}$ Annexin V-FITC and $5 \mu \mathrm{l}$ propidium iodide (PI) for $15 \mathrm{~min}$ in the dark at room temperature. The samples were detected by a Beckman Coulter FC500 cytometer immediately, and the FlowJo software version 10.4 (BD, United States) was used for data analysis.

\section{Measurement of Intracellular ROS Levels}

Intracellular ROS levels were measured by Reactive Oxygen Species Assay Kit (Beyotime Biotechnology, S0033S, China). Intracellular ROS is able to oxidize $2^{\prime}, 7^{\prime}$-dichlorofluoresceindiacetate (DCFH-DA) to fluorescent dichlorofluorescein (DCF) to quantify the level of ROS. The keratinocytes were planted to 6well plates and incubated with DCFH-DA at $37^{\circ} \mathrm{C}$ for $20 \mathrm{~min}$. The cells were washed three times with Dulbecco's phosphate buffered saline (DPBS) (Gibco, 14190144, United States). The fluorescence signal intensity and the percentages of positive probe were measured by a Beckman Coulter FC500 cytometer (Beckman, United States) and observed by fluorescence microscope (Leica, DMi8, Germany).

\section{Colony Formation Assay}

For colony formation assay, the infected keratinocytes were harvested and washed three times with DPBS. Each sample was added with $1 \mathrm{ml}$ of cold sterile water and placed on ice for $30 \mathrm{~min}$ allowing intracellular $S$. aureus to release. Diluent cell lysate was inoculated on MSA plate at $37^{\circ} \mathrm{C}$ for $24 \mathrm{~h}$. The number of CFU was counted by counting the number of bacterial communities. The experiment was repeated at least three times.

\section{Statistical Analyses}

All data were analyzed by SPSS Statistics 26.0 software (IBM, United States) and GraphPad Prism software (GraphPad Software, CA). Copmarison to statistical analysis was conducted using one-way ANOVA or LSD test and $p<0.05$ was considered statistically significant.

\section{RESULTS}

\section{IFN- $\lambda 1$ can Increase IL-28RA Expression in Keratinocytes Upon Staphylococcus aureus-Infection}

To illustrate whether IFN- $\lambda 1$ treatment could stimulate endogenous IFN- $\lambda 1$ secretion, we studied the expression of IFN- $\lambda 1$ and IL-28R (IL-28RA and IL-10RB) by qPCR and IL28RA by western blotting. The keratinocytes were pretreated with IFN- $\lambda 1$ for $24 \mathrm{~h}$, followed by $S$. aureus infection. Low concentration of IFN $\lambda 1(1 \mathrm{ng} / \mathrm{ml}$ and $10 \mathrm{ng} / \mathrm{ml})$ treatment significantly enhanced IL-28RA mRNA and protein expression in infected keratinocytes $(p<0.05)$, while high concentration of
IFN- $\lambda 1(100 \mathrm{ng} / \mathrm{ml})$ showed no effect (Figures 1A,B). The endogenous IFN- $\lambda 1$ and the accessory chain (IL-10RB) mRNA expression remained unchanged (Figure 1A).

\section{IFN- $\lambda 1$ Induces FLG Gene Expression and Inhibits TSLP in Human Keratinocytes During Staphylococcus aureus Infection}

FLG and IVL gene expression were evaluated in human keratinocytes. To eliminate the confounding factors irrelevant to keratinocyte proliferation, we performed cell proliferation assay. IFN- $\lambda 1$ of 1,10 and $100 \mathrm{ng} / \mathrm{ml}$ had no effect on cell proliferation (Figures $2 \mathrm{~A}-\mathrm{C}$ ). S. aureus infection induced IVL and FLG mRNA significant downregulation without marked cell death $(p<0.05)$ (Figures 2D, 3A). In non-infection model, IFN$\lambda 1100 \mathrm{ng} / \mathrm{ml}$ increased IVL mRNA expression (Figure 3A). In infection model, keratinocytes exposed to 10 and $100 \mathrm{ng} / \mathrm{ml}$ IFN$\lambda 1$ for $24 \mathrm{~h}$ induced significant FLG mRNA expression (Figure 3A).

Staphylococcus aureus infection induced significant elevation in TSLP level $(p<0.05)$ (Figure 3B). Pre-treatment of infected keratinocytes for $24 \mathrm{~h}$ with IFN- $\lambda 1$ significantly inhibited the TSLP level $(p<0.05)$ (Figure 3B).

\section{IFN- $\lambda 1$ Enhances Antimicrobial Peptide Expression in Human Primary Keratinocytes Upon Staphylococcus aureus Infection}

Keratinocytes were incubated for $24 \mathrm{~h}$ with IFN- $\lambda 1$ prior to $S$. aureus infection. In the non-infection model, the S100A7 mRNA expression was significantly elevated with increasing IFN- $\lambda 1$ concentration. S100A8 and S100A9 mRNA expression were increased in $10 \mathrm{ng} / \mathrm{ml}$ and $100 \mathrm{ng} / \mathrm{ml}$ IFN- $\lambda 1$ groups. hBD1 mRNA expression was up-regulated by high concentration of IFN- $\lambda 1(100 \mathrm{ng} / \mathrm{ml})$. hBD3 mRNA expression was increased in $10 \mathrm{ng} / \mathrm{ml}$ IFN $\lambda 1$ group (Figure $3 \mathrm{C}$ ). A substantial decrease in hBD1 and hBD3 mRNA expression were observed when cells were infected with $S$. aureus $(p<0.05)$ (Figure 3C). After infection, hBD1, hBD3 and S100A7 mRNA expression in IFN- $\lambda 110 \mathrm{ng} / \mathrm{ml}$ group were significantly increased. $\mathrm{hBD} 1$, S100A7, and S100A8 mRNA expression in IFN- $\lambda 1100 \mathrm{ng} / \mathrm{ml}$ group were also significantly increased $(p<0.05)$ (Figure 3C). hBD2 remained unchanged upon $S$. aureus in each group (Figure 3C).

\section{IFN- $\lambda 1$ Inhibits Staphylococcus aureus Colonization via IL-28RA-ROS-JAK-STAT1 Signaling Pathway in Human Primary Keratinocytes}

To study the effects of IFN- $\lambda 1$ on keratinocyte activation, we measured the ROS oxidase level which represents the microbicidal function of keratinocytes. IFN- $\lambda 1 \quad(10 \mathrm{ng} / \mathrm{ml})$ induced a substantial increase in IL-28RA expression infected keratinocytes $(p<0.05)$. IFN- $\lambda 1$ significantly increased mean fluorescence intensity (MFI) and the percentage of positive probe 


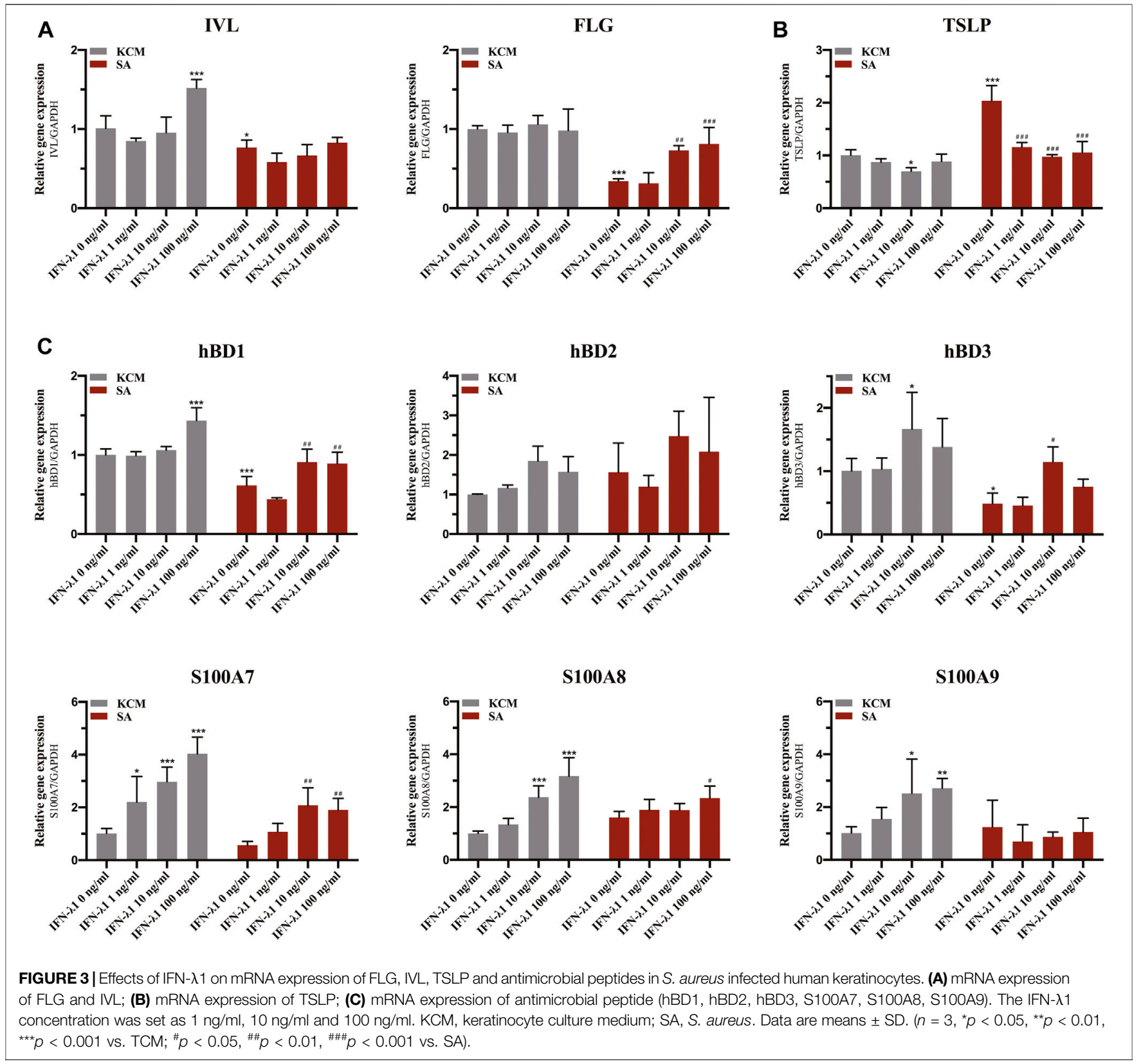

for intracellular ROS levels, regardless of $S$. aureus infection $(p<$ 0.05) (Figure 4A; Supplementary Figure S5A). Moreover, IFN$\lambda 1$ treatment significantly increased intracellular ROS levels and inhibited the attachment and the penetration of $S$. aureus (Figure 4C). S. aureus infection alone reduced MFI of ROS $(p<0.05)$ (Figure 4A). The level of pSTAT1 and STAT1 were significantly elevated by IFN- $\lambda 1$ treatment regardless of $S$. aureus infection $(p<0.05)$ (Figure 5A).

NADPH oxidase inhibitor (DPI) successfully blocked IFN- $\lambda 1$ induced antibacterial effects (Figure 4C). The MFI and the percentages of positive probe of ROS were down-regulated $(p<0.05)$ (Figure 4A, Supplementary Figure S5A). A four- fold decrease in PSTAT1 in keratinocytes was observed in the DPI group, compared to control (Figures 5A,B).

Experiments of transfection showed that siIL28RA-1 had the best knockdown ability and significantly inhibited IL28RA expression and pSTAT1/STAT1 level in keratinocytes $(p<0.05)$ (Supplementary Figures S4B,C). The knockdown of IL-28RA inhibited MFI and the percentages of positive probe of ROS without affecting $S$. aureus colonization $(p<$ 0.05) (Figures 4B,C; Supplementary Figure S5B). A fourteen-fold decrease in pSTAT1 in keratinocytes were observed in silL28RA-1 transfection group, compared to control (Figure 5B). 

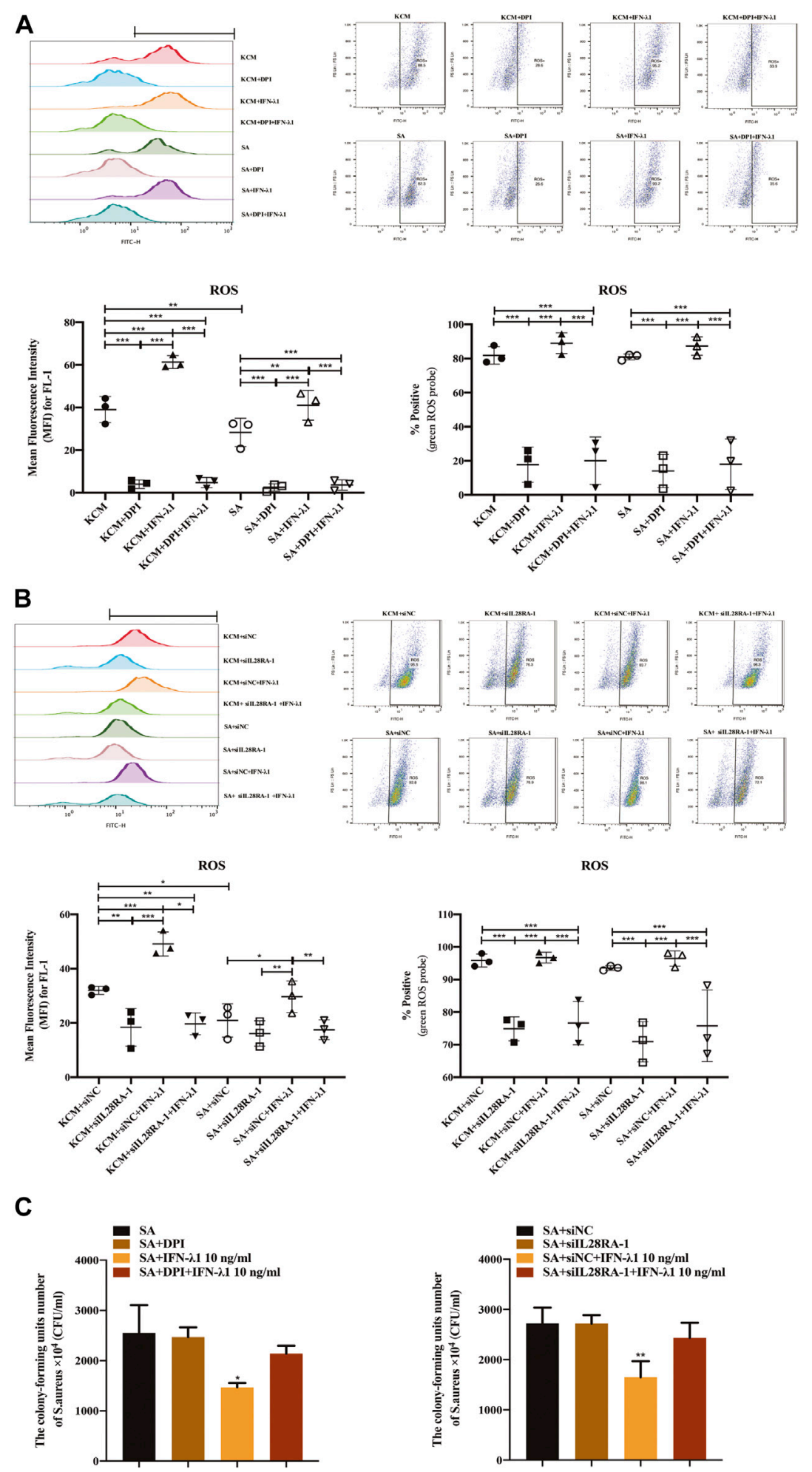

FIGURE 4 | IFN- $\lambda 1$ promotes ROS release and inhibits $S$. aureus colonization. (A,B) Effects of IFN- $\lambda 1$ on ROS expression in human keratinocytes by flow cytometry. (A) NADPH oxidase inhibitor (DPI) blocked ROS expression significantly; (B) Transfection of sill28RA-1 inhibited ROS expression significantly; (C) Inhibition of $S$. aureus colonization by IFN- $\lambda 1$. Keratinocytes infected with $S$. aureus were lyzed with sterile water. The lysates were plated on mannitol salt agar plates for $24 \mathrm{~h}$. The CFU was counted. ROS, reactive oxidase substrates; KCM, keratinocyte culture medium; SA, S. aureus; DPI, diphenyleneiodionium chloride; siNC, siRNA negative control; silL28RA-1, IL-28RA1 siRNA; CFU, colony forming units; Data are means \pm SD. $\left(n=3,{ }^{\star} p<0.05,{ }^{\star \star} p<0.01,{ }^{\star \star \star} p<0.001 \mathrm{vs} \mathrm{KCM}\right.$ or KCM + siNC; ${ }^{*} p<0.05$, $\# p<0.01, \# \# p<0.001$ vs. SA or SA + siNC). 


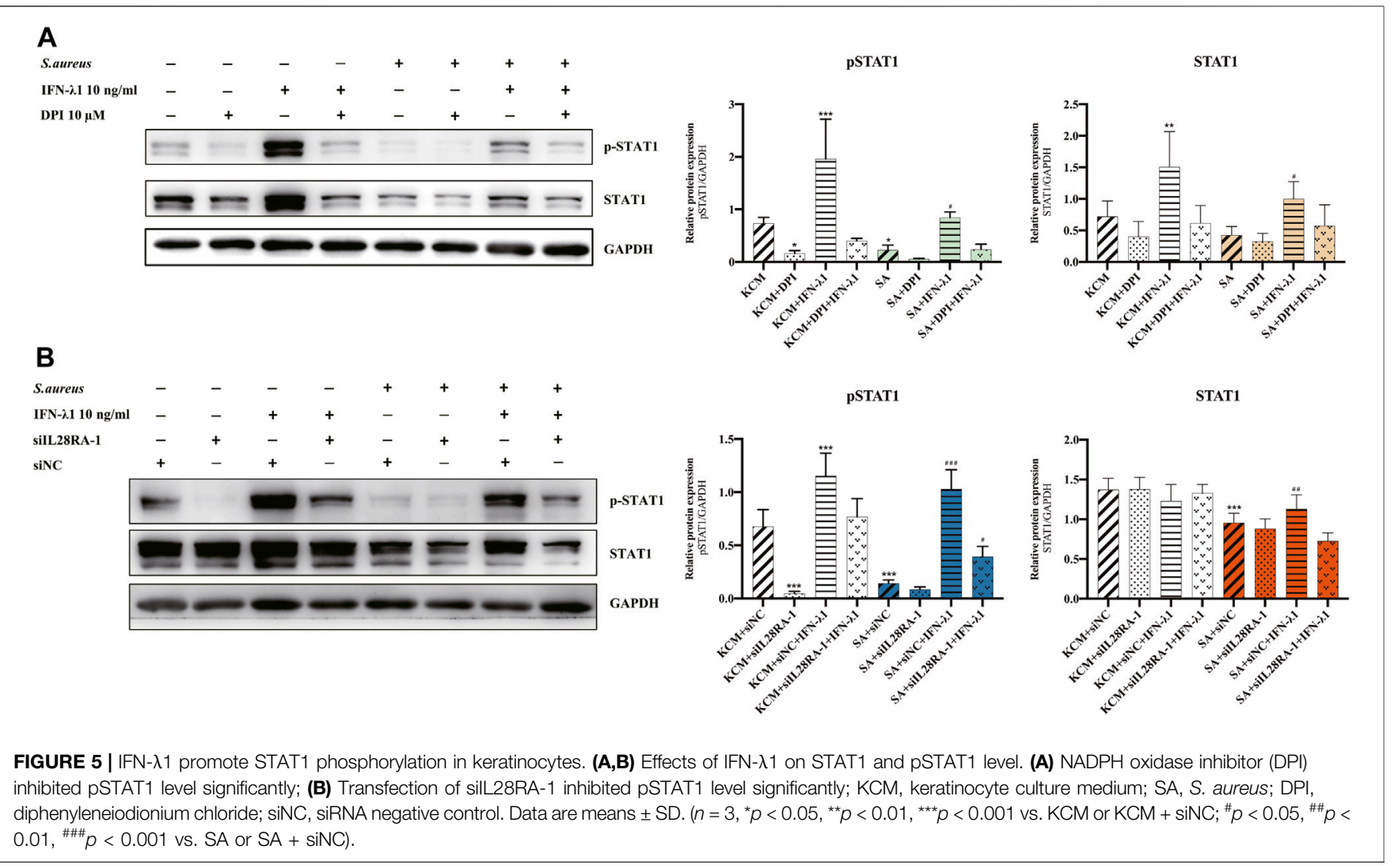

\section{DISCUSSION}

It is well-established that IFN- $\lambda$ represent an important frontline defense in barrier epithelia that fights against viral infections (Krug et al., 2020; Galani et al., 2021). Growing evidence has revealed new functions of IFN- $\lambda$, such as antibacterial, antifungal, and immunoregulatory functions. The effect of IFN- $\lambda 1$ on anti-S. aureus infection in the nasal mucosa and anti-Fetoplacental Listeriosis in placenta was also reported (Bierne et al., 2012; Lan et al., 2019). We studied the possible anti-bacterial role of IFN- $\lambda 1$ in human keratinocytes. We found that IFN- $\lambda 1$ could enhance the skin barrier gene and APM gene in keratinocytes exposed to $S$. aureus, keeping in accordance with the bacterial clearance function of the epidermal barrier. IFN- $\lambda 1$ can also regulate TSLP expression in keratinocytes infected by $S$. aureus to keratinocytes. Our study also demonstrated that the antimicrobial effects of IFN- $\lambda 1$ in keratinocytes might be via inhibition of attachment and penetration of $S$. aureus. In addition, IFN- $\lambda 1$ enhanced ROS production and STAT1 phosphorylation in keratinocytes irrespective of the presence of $S$. aureus. IL-28RA knockdown and ROS inhibition significantly inhibited the STAT1 phosphorylation. We hypothesize that IFN- $\lambda 1$ might perform its anti-bacterial function by 1 ) restoring the epidermis barrier function and modulating keratinocyte inflammation; 2) inhibiting $S$. aureus colonization via the IL-28R-ROS-JAKSTAT1 signaling pathway.
Skin is of importance for protecting the body from external microbial invasion and allergen stimulation. Previous studies demonstrated that Th2-type cytokines can promote $S$. aureus colonization in $\mathrm{AD}$ skin due to impaired skin barrier function and reduced production of antimicrobial peptide (Callewaert et al., 2020; Leung et al., 2020). Other studies also suggested that impaired skin barrier contributed to childhood asthma, food allergy, and allergic rhinosinusitis, indicating the importance to restore the skin barrier function in this disease (van den Oord and Sheikh, 2009). The relationship between IFN- $\lambda$ s and epithelial barrier protection in various organs, e.g. respiratory tract, lungs, and gastrointestinal tract had been well-established (Lozhkov et al., 2020; Stanifer et al., 2020). The ex vivo infection model showed that the barrier gene FLG and IVL were generally inhibited in $S$. aureus infection model. We focused on the barrier repair function of keratinocytes in $S$. aureus infection model and displayed that IFN- $\lambda 1$ could promote FLG expression in infected keratinocytes. This finding suggests that IFN- $\lambda 1$ may play a role in barrier function repairment by upregulating the FLG expression, thereby protecting the epidermis from invasion and colonization of $S$. aureus.

It is known that Th2-type cytokines (IL-4, IL-5, and IL-13) and epithelial-derived innate type-2 cytokines (TSLP) increased in skin lesions of acute stage of $\mathrm{AD}$ (DeVore et al., 2020; Edslev et al., 2020; Langan et al., 2020). TSLP is crucial for regulating the downstream IL-4/IL-13 and Th2 
differentiation. In addition, $S$. aureus inhibited STAT1 phosphorylation and contributed to downregulation of CXCL9 and CXCL10 in monocytes (Li et al., 2017). It has been reported that IFN- $\lambda$ s can inhibit Th 2 cytokine production in human peripheral blood mononuclear cells (PBMCs) (Jordan et al., 2007; Srinivas et al., 2008) and stimulate Th1 chemokines (CXCL9, CXCL10 and CXCL11) production in HaCaT cells (Syedbasha and Egli, 2017; Goel et al., 2020). In addition, IFN- $\lambda 2 / 3$ can inhibit TSLP secretion in bronchoalveolar lavage fluid of asthmatic mice (Won et al., 2019). Our findings indicate that IFN $-\lambda 1$ can also inhibt the secretion of TSLP, which might contribute to the alleviation of skin inflammation.

Skin lesions of $\mathrm{AD}$ are routinely associated with microbial colonization (S. aureus and HSV). The diminished expression of IFN- $\lambda 1$ and AMP was found in skin lesions of $\mathrm{AD}$ (Ong et al., 2002; de Jongh et al., 2005; Wolk et al., 2013). Bin et al. (Bin et al., 2014) reported that IFN- $\lambda 1$ was downregulated in HSV-1 infected PBMCs and in culture supernatants of PBMC of eczema herpeticum. This phenomenon was absent in $\mathrm{AD}$ patients without eczema herpeticum and in normal controls. We found that IFN- $\lambda 1$ can affect the AMP expression in keratinocytes infected with $S$. aureus. We also found the anti-bacterial function of IFN- $\lambda 1$ was via IL-28R-ROS-JAK-STAT signaling pathway. This was supported by increasing ROS levels and STAT1 phosphorylation in infected keratinocytes by IFN- $\lambda 1$ treatment.

In conclusion, we found that IFN- $\lambda 1$ facilitates the clearance of $S$. aureus in human epidermis keratinocytes through the IL28RA-ROS-JAK-STAT1 pathway. It also impacted the skin barrier gene and AMP secretion, and inhibited TSLP expression. This might contribute to antibacterial function of epidermis keratinocytes and reduction of skin inflammation.

\section{REFERENCES}

Ahn, K., Kim, B. E., Kim, J., and Leung, D. Y. (2020). Recent advances in atopic dermatitis. Curr. Opin. Immunol. 66, 14-21. doi:10.1016/j.coi.2020.02.007

Alduraywish, S. A., Lodge, C. J., Campbell, B., Allen, K. J., Erbas, B., Lowe, A. J., et al. (2016). The march from early life food sensitization to allergic disease: a systematic review and meta-analyses of birth cohort studies. Allergy 71, 77-89. doi:10.1111/all.12784

Banos-Lara Mdel, R., Harvey, L., Mendoza, A., Simms, D., Chouljenko, V. N., Wakamatsu, N., et al. (2015). Impact and regulation of lambda interferon response in human metapneumovirus infection. J. Virol. 89, 730-742. doi:10. 1128/JVI.02897-14

Bierne, H., Travier, L., Mahlakoiv, T., Tailleux, L., Subtil, A., Lebreton, A., et al. (2012). Activation of type III interferon genes by pathogenic bacteria in infected epithelial cells and mouse placenta. PLoS One 7, e39080. doi:10.1371/journal. pone.0039080

Bin, L., Edwards, M. G., Heiser, R., Streib, J. E., Richers, B., Hall, C. F., et al. (2014). Identification of novel gene signatures in patients with atopic dermatitis complicated by eczema herpeticum. J. Allergy Clin. Immunol. 134, 848-855. doi:10.1016/j.jaci.2014.07.018

Bin, L., Kim, B. E., Brauweiler, A., Goleva, E., Streib, J., Ji, Y., et al. (2012). Staphylococcus aureus alpha-toxin modulates skin host response to viral infection. J. Allergy Clin. Immunol. 130, 683-691.e2. doi:10.1016/j.jaci.2012. 06.019

\section{DATA AVAILABILITY STATEMENT}

The original contributions presented in the study are included in the article/Supplementary Material, further inquiries can be directed to the corresponding author.

\section{AUTHOR CONTRIBUTIONS}

$\mathrm{XW}, \mathrm{YZ}$ and $\mathrm{YG}$ performed the experiments and prepared diagrams. XW and XJW analyzed the data. XW and KL wrote the manuscript. XW and JZ designed the project. JZ supervised the project. JZ and KL provided financial support.

\section{FUNDING}

This work was supported by National Nature Science Foundation of China (No. 81903211).

\section{ACKNOWLEDGMENTS}

We thank Cong Huang (Peking University Shenzhen Hospital, Shenzhen, China) for providing human primary keratinocytes and Longyang Jin (Peking University People's Hospital, Beijing, China) for kindly providing the $S$. aureus strain.

\section{SUPPLEMENTARY MATERIAL}

The Supplementary Material for this article can be found online at: https://www.frontiersin.org/articles/10.3389/fphar.2021.652302/ full\#supplementary-material.

Broggi, A., Granucci, F., and Zanoni, I. (2020). Type III interferons: balancing tissue tolerance and resistance to pathogen invasion. J. Exp. Med. 217, e20190295. doi:10.1084/jem.20190295

Byrd, A. L., Deming, C., Cassidy, S. K. B., Harrison, O. J., Ng, W. I., Conlan, S., et al. (2017). Staphylococcus aureus and Staphylococcus epidermidis strain diversity underlying pediatric atopic dermatitis. Sci. Transl Med. 9, eaal4651. doi:10. 1126/scitranslmed.aal4651

Callewaert, C., Nakatsuji, T., Knight, R., Kosciolek, T., Vrbanac, A., Kotol, P., et al. (2020). IL-4Ralpha blockade by dupilumab decreases Staphylococcus aureus colonization and increases microbial diversity in atopic dermatitis. J. Invest. Dermatol. 140, 191-202.e197. doi:10.1016/j.jid.2019.05.024

de Jongh, G. J., Zeeuwen, P. L., Kucharekova, M., Pfundt, R., van der Valk, P. G., Blokx, W., et al. (2005). High expression levels of keratinocyte antimicrobial proteins in psoriasis compared with atopic dermatitis. J. Invest. Dermatol. 125, 1163-1173. doi:10.1111/j.0022-202X.2005.23935.x

DeVore, S. B., Gonzalez, T., Sherenian, M. G., Herr, A. B., and Khurana Hershey, G. K. (2020). On the surface: skin microbial exposure contributes to allergic disease. Ann. Allergy Asthma Immunol. 125, 628-638. doi:10.1016/j.anai.2020. 08.025

Edslev, S. M., Agner, T., and Andersen, P. S. (2020). Skin microbiome in atopic dermatitis. Acta Derm Venereol. 100, adv00164. doi:10.2340/00015555-3514

Eyerich, K., and Novak, N. (2013). Immunology of atopic eczema: overcoming the Th1/Th2 paradigm. Allergy 68, 974-982. doi:10.1111/all.12184

Galani, I. E., Rovina, N., Lampropoulou, V., Triantafyllia, V., Manioudaki, M., Pavlos, E., et al. (2021). Untuned antiviral immunity in COVID-19 revealed by 
temporal type I/III interferon patterns and flu comparison. Nat. Immunol. 22, 32-40. doi:10.1038/s41590-020-00840-x

Geoghegan, J. A., Irvine, A. D., and Foster, T. J. (2018). Staphylococcus aureus and atopic dermatitis: a complex and evolving relationship. Trends Microbiol. 26, 484-497. doi:10.1016/j.tim.2017.11.008

Goel, R. R., Wang, X., O’Neil, L. J., Nakabo, S., Hasneen, K., Gupta, S., et al. (2020). Interferon lambda promotes immune dysregulation and tissue inflammation in TLR7-induced lupus. Proc. Natl. Acad. Sci. U S A. 117, 5409-5419. doi:10.1073/ pnas. 1916897117

Jordan, W. J., Eskdale, J., Boniotto, M., Rodia, M., Kellner, D., and Gallagher, G. (2007). Modulation of the human cytokine response by interferon lambda-1 (IFN-lambda1/IL-29). Genes Immun. 8, 13-20. doi:10.1038/sj.gene.6364348

Kotenko, S. V., Gallagher, G., Baurin, V. V., Lewis-Antes, A., Shen, M., Shah, N. K., et al. (2003). IFN-lambdas mediate antiviral protection through a distinct class II cytokine receptor complex. Nat. Immunol. 4, 69-77. doi:10.1038/ni875

Krug, J., Kiefer, A., Koelle, J., Vuorinen, T., Xepapadaki, P., Stanic, B., et al. (2020). TLR 7/8 regulates type I and type III interferon signalling in RV1b induced allergic asthma. Eur. Respir. J. 57 (3), 2001562. doi:10.1183/13993003.01562-2020

Lan, F., Zhong, H., Zhang, N., Johnston, S. L., Wen, W., Papadopoulos, N., et al. (2019). IFN-lambdal enhances Staphylococcus aureus clearance in healthy nasal mucosa but not in nasal polyps. J. Allergy Clin. Immunol. 143, 1416-1425.e1414. doi:10.1016/j.jaci.2018.09.041

Langan, S. M., Irvine, A. D., and Weidinger, S. (2020). Atopic dermatitis. Lancet 396, 345-360. doi:10.1016/S0140-6736(20)31286-1

Lazear, H. M., Nice, T. J., and Diamond, M. S. (2015). Interferon-lambda: immune functions at barrier surfaces and beyond. Immunity 43, 15-28. doi:10.1016/j. immuni.2015.07.001

Leung, D. Y. M., Berdyshev, E., and Goleva, E. (2020). Cutaneous barrier dysfunction in allergic diseases. J. Allergy Clin. Immunol. 145, 1485-1497. doi:10.1016/j.jaci.2020.02.021

Li, Z., Levast, B., and Madrenas, J. (2017). Staphylococcus aureus downregulates IP10 production and prevents Th1 cell recruitment. J. Immunol. 198, 1865-1874. doi:10.4049/jimmunol.1601336

Lozhkov, A. A., Klotchenko, S. A., Ramsay, E. S., Moshkoff, H. D., Moshkoff, D. A., Vasin, A. V., et al. (2020). The key roles of interferon lambda in human molecular defense against respiratory viral infections. Pathogens 9, 989. doi:10. 3390/pathogens 9120989

Maher, S. G., Sheikh, F., Scarzello, A. J., Romero-Weaver, A. L., Baker, D. P., Donnelly, R. P., et al. (2008). IFNalpha and IFNlambda differ in their antiproliferative effects and duration of JAK/STAT signaling activity. Cancer Biol. Ther. 7, 1109-1115. doi:10.4161/cbt.7.7.6192

Marukian, S., Andrus, L., Sheahan, T. P., Jones, C. T., Charles, E. D., Ploss, A., et al. (2011). Hepatitis C virus induces interferon-lambda and interferon-stimulated genes in primary liver cultures. Hepatology 54, 1913-1923. doi:10.1002/hep.24580

Matsumoto, K., Iikura, K., Morita, H., and Saito, H. (2020). Barrier dysfunction in the atopic march-how does atopic dermatitis lead to asthma in children? J. Allergy Clin. Immunol. 145, 1551-1553. doi:10.1016/j.jaci.2020.04.014

Motala, C., Potter, P. C., Weinberg, E. G., Malherbe, D., and Hughes, J. (1986). Anti-Staphylococcus aureus-specific IgE in atopic dermatitis. J. Allergy Clin. Immunol. 78, 583-589. doi:10.1016/0091-6749(86)90075-8

Ong, P. Y., Ohtake, T., Brandt, C., Strickland, I., Boguniewicz, M., Ganz, T., et al. (2002). Endogenous antimicrobial peptides and skin infections in atopic dermatitis. N. Engl. J. Med. 347, 1151-1160. doi:10.1056/NEJMoa021481

Orfali, R. L., Yoshikawa, F. S. Y., Oliveira, L. M. D. S., Pereira, N. Z., de Lima, J. F., Ramos, Y. Á. L., et al. (2019). Staphylococcal enterotoxins modulate the effector CD4(+) T cell response by reshaping the gene expression profile in adults with atopic dermatitis. Sci. Rep. 9, 13082. doi:10.1038/s41598-019-49421-5

Planet, P. J., Parker, D., Cohen, T. S., Smith, H., Leon, J. D., Ryan, C., et al. (2016). Lambda interferon restructures the nasal microbiome and increases susceptibility to Staphylococcus aureus superinfection. mBio 7, e01939-15. doi:10.1128/mBio.01939-15

Sheppard, P., Kindsvogel, W., Xu, W., Henderson, K., Schlutsmeyer, S., Whitmore, T. E., et al. (2003). IL-28, IL-29 and their class II cytokine receptor IL-28R. Nat. Immunol. 4, 63-68. doi:10.1038/ni873

Sommereyns, C., Paul, S., Staeheli, P., and Michiels, T. (2008). IFN-lambda (IFNlambda) is expressed in a tissue-dependent fashion and primarily acts on epithelial cells in vivo. PLoS Pathog. 4, e1000017. doi:10.1371/journal.ppat. 1000017

Srinivas, S., Dai, J., Eskdale, J., Gallagher, G. E., Megjugorac, N. J., and Gallagher, G. (2008). Interferon-lambda1 (interleukin-29) preferentially down-regulates interleukin-13 over other $\mathrm{T}$ helper type 2 cytokine responses in vitro. Immunology 125, 492-502. doi:10.1111/j.1365-2567.2008.02862.x

Stanifer, M. L., Guo, C., Doldan, P., and Boulant, S. (2020). Importance of type I and III interferons at respiratory and intestinal barrier surfaces. Front. Immunol. 11, 608645. doi:10.3389/fimmu.2020.608645

Syedbasha, M., and Egli, A. (2017). Interferon lambda: modulating immunity in infectious diseases. Front. Immunol. 8, 119. doi:10.3389/fimmu.2017.00119

Tay, A. S. L., Li, C., Nandi, T., Chng, K. R., Andiappan, A. K., Mettu, V. S., et al. (2020). Atopic dermatitis microbiomes stratify into ecologic dermotypes enabling microbial virulence and disease severity. J. Allergy Clin. Immunol. 145 (6), 1517-1528. doi:10.1016/j.jaci.2020.09.031

Totte, J. E., van der Feltz, W. T., Hennekam, M., van Belkum, A., van Zuuren, E. J., and Pasmans, S. G. (2016). Prevalence and odds of Staphylococcus aureus carriage in atopic dermatitis: a systematic review and meta-analysis. $\mathrm{Br}$. J. Dermatol. 175, 687-695. doi:10.1111/bjd.14566

van den Oord, R. A., and Sheikh, A. (2009). Filaggrin gene defects and risk of developing allergic sensitisation and allergic disorders: systematic review and meta-analysis. BMJ 339, b2433. doi:10.1136/bmj.b2433

Weidinger, S., and Novak, N. (2016). Atopic dermatitis. Lancet 387, 1109-1122. doi:10.1016/S0140-6736(15)00149-X

Witte, K., Gruetz, G., Volk, H. D., Looman, A. C., Asadullah, K., Sterry, W., et al. (2009). Despite IFN-lambda receptor expression, blood immune cells, but not keratinocytes or melanocytes, have an impaired response to type III interferons: implications for therapeutic applications of these cytokines. Genes Immun. 10, 702-714. doi:10.1038/gene.2009.72

Wolk, K., Witte, K., Witte, E., Raftery, M., Kokolakis, G., Philipp, S., et al. (2013). IL-29 is produced by $\mathrm{T}(\mathrm{H}) 17$ cells and mediates the cutaneous antiviral competence in psoriasis. Sci. Transl Med. 5, 204ra129. doi:10.1126/ scitranslmed.3006245

Won, J., Gil, C. H., Jo, A., and Kim, H. J. (2019). Inhaled delivery of Interferonlambda restricts epithelial-derived Th2 inflammation in allergic asthma. Cytokine 119, 32-36. doi:10.1016/j.cyto.2019.02.010

Zitzmann, K., Brand, S., Baehs, S., Goke, B., Meinecke, J., Spottl, G., et al. (2006). Novel interferon-lambdas induce antiproliferative effects in neuroendocrine tumor cells. Biochem. Biophys. Res. Commun. 344, 1334-1341. doi:10.1016/j. bbrc.2006.04.043

Conflict of Interest: The authors declare that the research was conducted in the absence of any commercial or financial relationships that could be construed as a potential conflict of interest.

Copyright $(2021 \mathrm{Wu}$, Zhao, Gu, Li, Wang and Zhang. This is an open-access article distributed under the terms of the Creative Commons Attribution License (CC BY). The use, distribution or reproduction in other forums is permitted, provided the original author(s) and the copyright owner(s) are credited and that the original publication in this journal is cited, in accordance with accepted academic practice. No use, distribution or reproduction is permitted which does not comply with these terms. 